\title{
Preventing Chronic Diseases After Complicated Pregnancies in the COVID-19 Era: a Call to Action for PCPs
}

\author{
Mara E. Murray Horwitz, MD, MPH ${ }^{\top}$, Rose L. Molina, MD, MPH², and Tracy A. Battaglia, \\ $M D, M P H^{l}$
}

'Women's Health Unit, Section of General Internal Medicine, Evans Department of Medicine, Boston Medical Center and Boston University School of Medicine, 801 Massachusetts Avenue, Crosstown 1, Boston, MA, USA; ${ }^{2}$ Division of Global and Community Health, Department of Obstetrics and Gynecology, Beth Israel Deaconess Medical Center, Boston, MA, USA.

J Gen Intern Med 36(7):2127-9

DOI: $10.1007 / \mathrm{s} 11606-021-06734-4$

(c) Society of General Internal Medicine 2021

$\mathrm{P}$ regnancy complications affect up to one in three pregnancies and are increasingly linked to future chronic disease. ${ }^{1}$ For example, preeclampsia is associated with a doubled risk of cardiovascular disease (CVD), ${ }^{1}$ the leading cause of death in the USA, and gestational diabetes is associated with a 7-fold increased risk of type 2 diabetes. $^{2}$ Guidelines recommend preventive care starting within the first postpartum year to address such pregnancy-related health risks. ${ }^{2,3}$ Unfortunately, the connections between pregnancy health and future health are often neglected. Only half of patients successfully transition to primary care in the year after a medically complicated pregnancy ${ }^{4}$; of those who do, a small minority receive care informed by their pregnancy complication. ${ }^{5}$ Missed opportunities to address pregnancy-related chronic disease risk not only threaten individuals' wellbeing; they perpetuate longterm health inequities. Black, Hispanic, and low-income patients experience a double burden with higher rates of pregnancy complications and lower rates of postpartum follow-up, when compared with non-Hispanic White or higher-income patients. ${ }^{4}$

As the coronavirus disease 2019 (COVID-19) pandemic disrupts health care delivery and access to primary care especially in low-income communities, ${ }^{6}$ pregnancy- and chronic disease-related inequities are expected to worsen. The pandemic has simultaneously ushered in an era of virtual medicine that presents new opportunities to deliver coordinated and equitable care for postpartum patients. Pregnancy-informed primary care is now more important-and more possible - than before. This Viewpoint discusses the "acute on chronic" $"$ need, barriers, and potential solutions for primary care providers (PCPs) to address pregnancy-related chronic disease risk during and beyond the COVID-19 pandemic.

Received December 11, 2020

Accepted March 16, 2021

Published online March 29, 2021
These priority areas represent critical opportunities across the health care delivery continuum: (1) care transitions, (2) primary care, (3) PCP education and training, and (4) research.

\section{CARE TRANSITIONS}

Transitioning from obstetric to primary care is the first step toward managing pregnancy-related chronic disease risk. Rates of primary care during the first postpartum year hover around $50-60 \%{ }^{4}$ These transition failures have been attributed to patient-, provider-, and system-level factors. First, the unique challenges of postpartum life (e.g., caring for a newborn, returning to work) make it difficult for patients to seek care in the months after pregnancy - exactly when follow-up is needed. ${ }^{2,3}$ Second, providers express confusion about who is responsible for, and what should be included in, care during the year after pregnancy. ${ }^{4,5}$ Poor cross-specialty communication, including inconsistent documentation, contributes to fragmented care. ${ }^{1,5}$ In addition, discontinuities in postpartum health insurance reduce or complicate access to care. This barrier is significant for low-income patients; in all 13 nonexpansion states, pregnancy-based Medicaid ends just 60 days after delivery. The ongoing pandemic further threatens postpartum care transitions through restrictions on in-person care, loss of jobs and employer-based insurance, and new competing demands for public funds. For instance, Tennessee is no longer expanding postpartum Medicaid, citing COVID-19related budget cuts.

Systematic planning for the postpartum care transition - supported by funding for community health centers $^{6}$ and universal health insurance for the entire year after pregnancy - is needed. Obstetricians and PCPs must work together to define roles and systematize handovers. Innovations in virtual health care delivery in the COVID-19 era can incorporate transition support after pregnancies. The electronic medical record (EMR) can be used to monitor postpartum follow-up, identify high-risk individuals, and automate patient outreach. For this to work, pregnancy complications must be reliably recorded in the EMR. In addition, numerous patientcentered interventions have been suggested to improve early postpartum visit attendance, and may also be effective for 
primary care follow-up; these include prenatal counseling, delivery discharge checklists, and patient navigation. ${ }^{2}$ Such programs should be implemented, and their impacts studied and disseminated.

\section{PRIMARY CARE}

Timely and longitudinal primary care is essential given that many pregnancy complications require regular follow-up for associated chronic disease risks, beginning within the year after pregnancy. ${ }^{2,}{ }^{3}$ For example, patients with prior preeclampsia or gestational diabetes may require annual CVD risk assessment ${ }^{3}$ or glycemic testing, ${ }^{2}$ respectively. Yet internists report low levels of comfort with pregnancy-related care. ${ }^{5}$ In one anonymous survey, only $9 \%$ of internists counseled patients with prior preeclampsia about their CVD risk. ${ }^{5}$ Half of patients with prior gestational diabetes undergo glycemic testing within the first postpartum year. ${ }^{4}$

For practicing PCPs, system-based interventions can address widespread gaps in comfort and experience. Checklists, templates, and best practice reminders can help PCPs to collect, document, and incorporate pregnancy complications into primary care management. ${ }^{1}$ In addition, telehealth and selfmonitoring tools (e.g., home blood pressure monitors) may be powerful ways to promote equity and agency in postpartum care when focused on patients with limited social and financial supports. ${ }^{2}$ These approaches should be deployed immediately, wherever possible, to offset the effects of the pandemic on healthcare access in low-income communities. ${ }^{6}$ Populationbased clinical registries are also urgently needed to monitor and support best practices in pregnancy follow-up, especially over time as methods of risk assessment ${ }^{1}$ and management evolve in this rapidly changing field.

\section{PCP EDUCATION AND TRAINING}

Prevention of chronic diseases and health inequities requires a competent workforce. Yet directors of Internal Medicine residency programs - which produce the majority of primary care physicians in the USA - consistently rank pregnancy-related topics as lowest priority in "women's health."7 They also report a shortage of relevant training opportunities, ${ }^{7}$ no doubt both a part and a product of the problem. Furthermore, comprehensive guidance for PCPs on pregnancy-related chronic disease risk management care is lacking.

For future PCPs, medical school and residency curricula must teach about the obstetric history as an essential consideration in chronic disease care. By partnering with Obstetrics \& Gynecology and Family Medicine departments, Internal Medicine programs can expand their training opportunities and build bridges for better care coordination. Practical guidance for PCPs on long-term pregnancy-related chronic disease risk management is urgently needed.

\section{HEALTH CARE DELIVERY RESEARCH}

Barriers to care after pregnancy are complex, and are likely to require multi-level solutions tailored to diverse contexts. Community Care of North Carolina is a model program ${ }^{2}$; it supports care coordination and chronic disease risk management through a combination of patient-, provider-, and system-level interventions, tailored to individual risk levels, and applied throughout the prenatal to postpartum care pathway for Medicaid patients.

Population-specific research is needed to understand barriers and facilitators to primary care after pregnancy, and to develop and pilot test interventions to address them. In addition, the COVID-19 pandemic has been a "natural experiment" in care delivery; its impact on PCPs' ability to provide equitable pregnancy-informed care should be studied. Implementation science can help scale locally effective interventions into widespread practice improvements.

\section{CONCLUSIONS}

Critical areas of opportunities exist for primary care leaders to improve long-term outcomes for patients with pregnancy complications. COVID-19 has increased the urgency of this work, by potentiating a future pandemic of preventable chronic diseases. PCPs must work with obstetric care providers to ensure smooth and systematic postpartum care transitions; incorporate obstetric history into chronic disease risk assessment and management; and teach and train future PCPs to do the same. Virtual health innovations in the COVID-19 era can be used to support postpartum care transitions. Funding for community health centers and extended health insurance for all postpartum patients are essential to these efforts. In addition, research is needed to develop and disseminate multi-level interventions (e.g., patient self-monitoring tools, EMR-based clinician support, and population health management) that improve PCPs' ability to prevent chronic diseases and longterm health inequities arising from complicated pregnancies.

Corresponding Author: Mara E. Murray Horwitz, MD, MPH; Women's Health Unit, Section of General Internal Medicine, Evans Department of Medicine, Boston Medical Center and Boston University School of Medicine, 801 Massachusetts Avenue, Crosstown 1, Boston, MA 02118, USA (e-mail: Mara.MurrayHorwitz@bmc.org).

\section{Declarations:}

Conflict of Interest: The authors declare that they do not have a conflict of interest.

\section{REFERENCES}

1. Okoth K, Chandan JS, Marshall T, et al. Association between the reproductive health of young women and cardiovascular disease in later life: umbrella review. BMJ. 2020; m3502. https://doi.org/10.1136/bmj. m3502.

2. American College of Obstetricians and Gynecologists. ACOG Postpartum Toolkit. Published online 2018. https://www.acog.org/-/media/project/ 
acog/acogorg/files/pdfs/publications/2018-postpartum-toolkit.pdf? la=en\&hash=0A51794942480674F3376F6E974EA58C. Accessed August $1,2020$.

3. Bushnell C, McCullough LD, Awad IA, et al. Guidelines for the prevention of stroke in women: a statement for healthcare professionals from the American Heart Association/American Stroke Association. Stroke. 2014;45(5): 1545-1588. https://doi.org/10.1161/01.str.0000442009. 06663.48.

4. Walker LO, Murphey CL, Nichols F. The broken thread of health promotion and disease prevention for women during the postpartum period. J Perinat Educ. 2015;24(2):81-92. https://doi.org/10.1891/10581243.24.2.81.

5. Roth H, LeMarquand G, Henry A, Homer C. Assessing knowledge gaps of women and healthcare providers concerning cardiovascular risk after hypertensive disorders of pregnancy - a scoping review. Front Cardiovasc Med. 2019;6. https://doi.org/10.3389/fcvm.2019.00178.

6. Wen LS, Sadeghi NB. Addressing racial health disparities in the COVID-19 pandemic: immediate and long-term policy solutions. Health Affairs Blog. 2020. https://www.healthaffairs.org/do/10.1377/hblog20200716. 620294/full/. Accessed September 22, 2020

7. Casas RS, Hallett LD, Rich CA, Gerber MR, Battaglia TA. Program directors' perceptions of resident education in women's health: a national survey. J Womens Health. 2016;26(2):133-140. https://doi.org/10.1089/ jwh.2016.5860.

Publisher's Note: Springer Nature remains neutral with regard to jurisdictional claims in published maps and institutional affiliations. 